\title{
Horizons/Théâtre
}

Revue d'études théâtrales

\section{Le Doozi, chœur rythmique du Didiga de Zadi Zaourou}

Lou Touboué Jacqueline Soupé

\section{OpenEdition}

1 Journals

Édition électronique

URL : https://journals.openedition.org/ht/1425

DOI : $10.4000 /$ ht. 1425

ISSN : 2678-5420

Éditeur

Presses universitaires de Bordeaux

\section{Édition imprimée}

Date de publication : 1 janvier 2019

Pagination : 44-58

ISBN : 979-10-300-0718-3

ISSN : 2261-4591

\section{Référence électronique}

Lou Touboué Jacqueline Soupé, « Le Doozi, chœur rythmique du Didiga de Zadi Zaourou », Horizons/ Théâtre [En ligne], 14 | 2019, mis en ligne le 01 juin 2022, consulté le 30 juin 2022. URL : http:// journals.openedition.org/ht/1425; DOI : https://doi.org/10.4000/ht.1425

Licence Creative Commons

La revue Horizons/Théâtre est mise à disposition selon les termes de la Licence Creative Commons Attribution - Pas d'Utilisation Commerciale - Pas de Modification 4.0 International. 


\section{LOU TOUBOUÉ JACQUeLINe SOUPÉ}

Soupé Lou Touboué Jacqueline est Maître-assistant au département de Lettres Modernes à I'Université Félix Houphouët Boigny de Cocody-Abidjan où elle enseigne le théâtre. Ses recherches portent sur le théâtre de Zadi Zaourou, le thème de la liberté et sur les formes $\mathrm{d}^{\prime}$ expressions dramatiques traditionnelles et contemporaines. Elle a publié plusieurs articles dont La dramaturgie du conte dans "La guerre des femmes " de Zadi Zaourou, Le personnage de l'agent rythmique dans "Les Sofas » et "Le secret des dieux » de Zadi Zaourou, Les mythes du résistant et des " pères de la nation » dans "Les Sofas » et "Le secret des dieux » de Zadi Zaourou, La complainte d'Ewadi, un cas de transfrontalité générique, La figure féminine dans le théâtre ivoirien ou la représentation de la femme combattante.

Mail: soupe_lou@yahoo.fr

Résumé : Si le théâtre est mimesis, spectacle, cette forme d'expression artistique existe dans l'Afrique traditionnelle. Ces arts spécifiques aux différentes régions et qui s'apparentent au théâtre ont pour nom : kotéba, mvêt, didiga, etc. Jouant un rôle social, économique, religieux, politique important, ces expressions dramatiques se faisaient, non pas par le théâtre tel que les Grecs vont l'expérimenter et plus tard toutes les sociétés occidentales. Il est, de ce point de vue, évident de les analyser, selon des critères esthétiques propres. Cependant, en tenant compte de l'histoire coloniale de l'Afrique et de l'avènement d'un monde moderne globalisé, il est nécessaire d'inscrire et d'appréhender aujourd'hui ces formes d'expressions

Abstract : If the theater is mimesis, show, this form of artistic expression exists in traditional Africa. These arts, which are specific to the different regions and which resemble the theater, are called koteba, mvet, didiga, etc. Playing a social, economic, religious and important political role, these dramatic expressions were made, not by the theater as the Greeks will experience it, and later by all Western societies. From this point of view, it is necessary to analyze them according to their own aesthetic criteria. However, taking into account the colonial history of Africa and the advent of a globalized modern world, it is necessary to inscribe and apprehend today these forms of dramatic expression in dramatiques dans une vision globalisante, dans un choix éclectique. Telle est en réalité la démarche de Zadi Zaourou qui reprend le didiga du pays bété ivoirien pour lui donner un écho plus important et le faire exprimer dans cette société de rencontres. Le didiga est un spectacle dramatique d'initiation, de quête, de l'entre deux, symbolisé par un des personnages récurrents, le Doozi, chœur rythmique du didiga. Parce que sa situation relève toujours de l'extraordinaire, du surréel, Zadi Zaourou, le théoricien du genre, le définit comme un art de l'impensable que cette contribution vise à décrypter..

Mots-Clés : Didiga, Doozi, art dramatiques, initiation, éclectique.

a globalizing vision, in an eclectic choice. This is really the approach of Zadi Zaourou who takes the didiga ivorian country to give a greater voice and make it express in this society of encounter. The didiga is a dramatic show of initiation, quest, in-between, symbolized by one of the recurring characters, the Doozi, didiga's rhythmic chorus. Because his situation is always the extraordinary, the surreal, Zadi Zaourou, the gender theorist defines it as an art of the unthinkable that this contribution aims to decrypt.

Keywords: Didiga, Doozi, drama, initiation, eclectic. 


\section{Le Doozi, chœur rythmique du Didiga de Zadi Zaourou}

\section{Introduction}

ABORDER LE THÉÂTRE IMPLIQUE LA QUESTION DU RELATIVISME culturel. Le mot théâtre désigne le lieu ou la scène, l'édifice, le spectacle et la littérature. Selon la définition qu'en donne Paul Aron ${ }^{1}$, le lieu est l'endroit où des acteurs jouent. L'édifice c'est le bâtiment ou le site à l'intérieur duquel se trouve la scène aménagée pour la manifestation spectaculaire. Le spectacle c'est « ce qui s'offre au regard ${ }^{2} \gg$. La littérature renvoie au texte qui formalise l'esthétique du genre. Ce théâtre s'inscrit dans une filiation, s'exprime conformément à une culture, une civilisation. Sa production obéit à des règles. Ses conditions de réception sont soumises à des codes. Aussi douloureux que cela puisse paraître, ce théâtre pris au sens européen du terme n'existe pas dans l'Afrique profonde comme le démontre Bernard Dadié dans Mon pays et son théâtre .

En revanche, selon Aristote, le théâtre est mimesis, c'est-à-dire l'imitation des actions humaines et naît en Grèce antique dans un contexte de « fêtes religieuses ${ }^{4} \gg$. Dans Poétique, il écrit : «On peut imiter en racontant ou on raconte par la bouche d'un autre, comme fait Homère, ou on garde sa personnalité sans la changer, ou en présentant tous les personnages comme agissant, comme "en actes" $\gg$. Aristote décrit ainsi comment par le jeu, le comédien peut faire parler son corps, son être entier pour traduire une situation donnée. Engagé dans les luttes socio-politiques en faveur de l'homme, Bertolt Brecht conçoit le théâtre comme un instrument de réflexion social dont la structure repose sur la narration. Pour Christian Biet et Christophe Triau, « Le théâtre est d'abord un spectacle, une performance éphémère, la prestation de comédiens devant des spectateurs qui regardent, un travail corporel, un exercice vocal et gestuel adressés le plus souvent dans un décor particulier ${ }^{6}$. $\gg$ Si l'on tient compte de ces conceptions aristotéliciennes, brechtienne et contemporaine, le théâtre est mimesis, action, spectacle, performance. Constituant l'ensemble des arts dramatiques, ce théâtre est inhérent à toutes les sociétés humaines. Les modalités de productions artistiques étant spécifiques à chaque 
groupe humain, à chaque mode de pensée et de fonctionnement, le théâtre tel que les Grecs l'ont imaginé et que les peuples européens ont en partage, existe partout sous diverses appellations et s'exprime conformément à l'âme des peuples. Il n'est donc pas possible qu'un art qui ressemble à ce théâtre soit totalement méconnu en Afrique avant la colonisation. Aussi, comme le nô japonais, le kathakali indien, le kotéba, le mvêt et le didiga traditionnels ne peuvent-ils être confondus avec le théâtre occidental.

Un constat s'impose cependant. Le théâtre tel qu'il est pratiqué aujourd'hui en Afrique est un héritage colonial transmis depuis l'École Primaire Supérieure de Bingerville. Depuis les indépendances, des générations d'auteurs tentent de réorienter les techniques scripturales et spectaculaires du genre. La tradition orale constitue une des sources d'inspiration de ces grandes figures de l'art dramatique africain dont fait partie Zadi Zaourou. Puisque l'histoire ne se réécrira pas, le didiga ancien qui relevait d'une certaine pratique culturelle précise est exhumé et systématisé dans le cadre du théâtre de recherche ivoirien pour prendre la forme d'un art du spectacle au centre duquel se trouve le personnage du Doozi. Ainsi s'explique l'intitulé de cette réflexion : Le Doozi, chour rythmique du didiga.

À partir de ce personnage présenté comme la métaphore du genre, ce sujet vise à montrer que le didiga est un art de la scène fondé essentiellement sur l'initiation. Trois pièces représentatives du didiga, Le secret des dieux, La guerre des femmes et La Termitière dont l'expérimentation donne naissance à une activité théâtrale entendue comme pratique et texte, modélise l'engagement littéraire et même politique de l'homme de lettres ivoirien. Compte tenu du fait que la société s'inscrit dans le didiga et le crée, ce sujet sera analysé sous l'angle de la sociocritique. Quelles sont les modalités de l'expression dramatique du didga ? Dans quelle mesure, Le Doozi est un moyen de structuration du didiga ? Quelle est l'essence du didiga ? Telles sont les interrogations qui vont structurer cette réflexion.

\section{Le didiga, un art total de la scène}

Même si le théâtre africain traditionnel n'existe pas sur le modèle occidental, il y a des formes d'expressions artistiques mimétiques, fonction de l'organisation de la société, qui étaient de type sacré ou profane. Le didiga fait partie de ces expressions dont le but est de former et divertir les populations. Zadi Zaourou situe son cadre conceptuel en ces termes :

Originellement, le concept de Didiga renvoie à un art de type particulier que pratiquaient les chasseurs bétés. À la base de cet art, un instrument de musique 
qui est un instrument parleur : dôdô, connu en français sous le nom d'arc musical. Les chasseurs au cours de longues randonnées en forêt [...] couraient de multiples et redoutables aventures. Lorsqu'ils rentraient au village, ces héros, qu'admirait le peuple parce qu'ils conjuraient la famine et symbolisaient le courage et l'abnégation, se plaisaient souvent à conter au public leurs merveilleuses équipées ${ }^{7}$.

Il en découle que le didiga est un art oratoire pratiqué par les chasseurs du pays bété d'où Zadi Zaourou est originaire. Dans l'œuvre du dramaturge cohabitent deux types de didiga : l'ancien, le traditionnel et le moderne, celui expérimenté par la Compagnie Didiga. Le didiga traditionnel a pour héros central Djerbeugbeu, un personnage symbolique représentant les chasseurs. Ce didiga est dit sous la forme de conte. Le chasseur-conteur relate les hauts faits, les combats épiques, les métamorphoses, les événements extraordinaires et insolites vécus réellement dans la jungle. À l'origine, le didiga est donc une mimesis définie comme un acte de reproduction. Le didiga n'est pas le théâtre. Il n'est pas non plus une structure préthéâtrale. Le didiga, c'est le didiga.

Cependant, son expression, les performances du chasseur-conteur en font un art mimétique qui peut, dans certaines circonstances, s'apparenter au jeu théâtral. Ce n'est donc pas parce que ce jeu ressemble au théâtre que le didiga est du théâtre. Le didiga est un art mimétique structuré par des récits qui peuvent être épiques, dramatiques, poétiques. C'est cet art complexe qui donne naissance au didiga moderne dont Zadi Zaourou situe ici les traits caractéristiques :

Le didiga moderne est né en 1980, avec la Compagnie qui porte son nom. Contrairement au didiga ancien, c'est un art de la scène. Comme l'ancien mais avec des différences de tous ordres, il se caractérise par les traits suivants : une situation, l'intrigue, relevant toujours de l'impensable. Un héros idéal et redresseur de torts, tout à l'image de l'antique héros de Didiga, Djergbeugbeu. Des affrontements épiques, des métamorphoses, des mystères... Bref, une action toute trépidante et chargée de vie parce que s'accomplissant dans la lutte de chaque instant. Une symbolique des couleurs [...] La présence d'un esprit, Ouga, $[\ldots]$ Un symbolisme des personnages, des situations, des sons, des objets, des lumières.

Il s'agit là des prémices de la poétique du didiga dont les éléments constitutifs servent non seulement à la fabrication des pièces mais également comme un ensemble de moyens d'interprétation scénique. Le didiga moderne prend la forme d'un spectacle théâtral soumis à un ensemble de conventions diverses 
et surtout, inscrites dans une vision totalitaire de l'art théorisée par Léopold Sédar Senghor dans Négritude et Humanisme. À ce propos, il écrit : « La musique est liée à la parole et à la danse. La musique ne peut se concevoir sans le geste, sans la danse. Ni la danse sans la peinture et la sculpture ${ }^{9}$. $\gg$ Pour le poète sénégalais, l'art africain se définit par un rapport évident d'interdépendance et d'unité des arts. À la suite de Senghor, et analysant L'anthropologie $d u$ théâtre négro-africain traditionnel ${ }^{10}$, Harris Memel-Fotê insiste sur la synthèse artistique, technique et poétique comme une caractéristique majeure de l'art dramatique africain. Dans un article en hommage posthume au chansonnier Srolou, Christophe Wondji consacre cette idée de perméabilité de l'art. Il affirme : «En Afrique, la chanson est à la foi littérature et musique, parole et danse, discours et rythme, pensée et expression corporelle ${ }^{11}$. » Cette interférence immanente des arts négro-africains révèle le didiga dans sa dimension symbolique, philosophique ou métaphysique sur lesquelles nous reviendrons.

L'hétérogénéité du didiga est le résultat de divers modes de transpositions. Dans le premier cas, le dramaturge ivoirien intègre les circonstances qui donnent lieu à des manifestations publiques ésotériques ou exotériques comme les rites d'initiation, les fêtes des moissons, les jeux, les funérailles, les cultes périodiques des ancêtres et des dieux, les rites de guerre, de sortie de masques, d'intronisation de chefs ou de toutes autres cérémonies solennelles. Successivement, des scènes comme les épreuves subies par le néophyte, l'hymne au travail, le jeu de la graine dans La Termitière; les funérailles de Zouzou, l'offrande de la jeune vierge, la guerre opposant la cité des femmes à celle des hommes dans La guerre des femmes ; la figure du Doozi, les apparitions du monarque dans Le secret des dieux sont divers faits et événements sociaux qu'il intègre comme des éléments faisant partie du jeu scénique. On est dans la mimique de la société, Zadi Zaourou représente la société telle quelle.

Second procédé utilisé pour faire s'exprimer le didiga, l'intergénéricité permet d'étudier la manière dont un genre naît d'un autre genre, comment un genre prend les habits d'un autre genre. Ici, le didiga naît du conte. Il emprunte les techniques du conte pour faire fonctionner le théâtre lui-même comme un conte. Ces techniques sont déployées dans les trois pièces qui relèvent du didiga. Zadi Zaourou organise ses pièces sous la forme de veillées de contes. Les textes donnent l'impression de commencer justement par un conte. Le cercle de conte est animé par le conteur principal et son agent rythmique au début. Médiateur entre l'émetteur et le récepteur, l'agent rythmique est l'animateur sur lequel repose la distribution de la parole et la saisie des 
échanges. Zadi Zaourou présente ce contexte de la circulation triadique de la parole dans le cas d'un spectacle de conte :

Observons le cercle de conte sur la place du village. Nous y découvrons le conteur, et généralement un deuxième personnage qui le suit comme son ombre et ponctue sa parole, et enfin le public chour. Nous retrouverons là l'espace triadique dont il a été question. - C'est en empruntant ce circuit ternaire que s'accomplira la parole du conte : celle du conteur, celle de son agent rythmique, celle du public enfin ${ }^{12}$.

Dans le cadre d'un cercle de conte spectacle, l'agent rythmique ponctue les propos du conteur principal. L'exemple le mieux abouti est le prologue de Le secret des dieux. Ces conteurs sont relayés ensuite par le héros ou un autre personnage, en général, le double ou l'alter ego du héros. Dans La guerre des femmes, Schéhérazade l'héroïne joue le rôle de conteuse à la suite du récitant. Le décor est planté à la fois dans la mise en abyme de ce qu'il y a un cercle de conte qui est établi mais à l'intérieur tel que le récit lui-même est organisé. Le temps et l'espace sont souvent mal déterminés. Les animaux, les esprits et les hommes dialoguent comme cela n'est souvent possible que dans les contes. Le découpage des pièces transgresse le mode de structuration classique : Le secret des dieux est une pièce composée en tableaux rythmiques et en tableaux comportant des titres ; La guerre des femmes en tableaux et en astérisques; La Termitière comporte des numéros avec des titres. L'abondance des didascalies narratives, sorte de voix auctoriales rappelle la performance du chasseur-conteur. Le décloisonnement générique apparaît sous la forme fonctionnelle dans la mesure où l'intergénéricité va au-delà du mélange de genres. Un texte de théâtre peut fonctionner comme un conte. C'est cette intergénéricité fonctionnelle qui permet à Zadi Zaourou de procéder à l'actualisation thématique en traitant des thèmes de la dictature, du pouvoir, de la liberté.

Le didiga se caractérise par l'intégration de la musique, une autre discipline artistique. Il a été reproché au dramaturge ivoirien de produire un théâtre dansé. C'est que, la musique et plus singulièrement la danse est un art mimétique. Dans la section de sa thèse consacrée aux formes de représentations dramatiques, Barthélémy Kotchy-N'Guessan ${ }^{13}$ montre que la danse est le point de départ de l'expression dramatique en Afrique. En tenant compte des valeurs artistiques et sémantiques des danses, il propose une taxinomie en cinq types à savoir les danses mimées, les danses à gestes-symboles, les danses à mimes et à gestes-symboles, les danses à personnages incarnés ou à symboles anthropomorphes et les danses à dramatisation complexe. Le di- 
diga prend en compte les danses mimées en représentant le Têmaté dans la première séquence intitulée «L'hymne au travail I » de La Termitière ainsi que le Klin-Kply ou l'Attougblan, une danse à gestes-symboles au tableau I titré «La parade » dans Le secret des dieux. Ces danses exécutées dans un cadre ludique précis ont un rapport avec les activités sociales et expriment des émotions collectives. La danse en elle-même est un art total qui va impliquer l'instrumentiste, le costumier, le chanteur, le masque, le danseur. Les danses africaines traditionnelles ont une dimension complètement théâtrale. En les intégrant dans son jeu dramatique, Zadi Zaourou montre que les Africains ont mimé la société à partir de la danse et non à partir d'une représentation théâtrale comme dans les sociétés occidentales. C'est pour cette raison qu'il y a des danses pour chacune des situations : la guerre, les naissances, les mariages, l'initiation, les nouvelles récoltes. À l'intérieur, il y a des compartiments, c'est-à-dire des danses pour les femmes, les enfants, les hommes. En fonction des circonstances en face desquelles se trouve la communauté, il y a une danse pour marquer cette situation particulière. Ceci est la preuve d'une capacité de théorisation du monde qui se résumait essentiellement dans la danse. Les manifestations culturelles étant rythmées par la danse, les Africains ont joué, représenté leur société par ce mode d'expression symbolisé ici par la figure du Doozi.

\section{Les figures du Doozi}

Personnage récurrent dans le didiga, Le Doozi est un danseur en initiation dont la construction et l'évolution riment avec des situations dramatiques précises. Au niveau linguistique, Le Doozi « désigne l'assistant du masque au moment où il entre en scène, pendant qu'il est en scène et au moment où il sort de la scène. C'est un danseur émérite qui est en initiation, un néophyte dont la vocation est de devenir un masque ${ }^{14} \gg$. À la base, Le Doozi est donc un artiste dont la double dimension se révèle dans sa construction même. Sa théâtralisation rappelle en effet le personnage de théâtre qui désignait à l'origine le masque porté par les acteurs sur scène et certains personnages de la vie traditionnelle comme le griot, le chasseur ayant une valeur théâtrale par leurs fonctions sociales. Ce double niveau de perception du Doozi en fait une figure majeure dans la fable dramatique et dans le dispositif esthétique du didiga. Le Doozi est identifié sous ce nom uniquement dans Le secret des dieux. Dans les deux autres pièces, la présence du Doozi relève purement de sa symbolisation ou de sa métaphorisation. 
Le Doozi est en réalité un assistant du masque, symbole de pouvoir. Autour du masque, il y a toute une organisation politique, spirituelle, culturelle. Zadi Zaourou recrée cet univers dans lequel le personnage joue son propre rôle d'assistant. Le Doozi est au cœur du pouvoir politique en tant que ministre du roi Edoukou. Entrer dans ce cercle signifie que Le Doozi a acquis une certaine stature dans le domaine de l'initiation. Il est en initiation ou il est initié. Le dramaturge se sert de ce rôle pour actualiser la thématique du pouvoir, ficeler les mécanismes dramatiques dont les différentes métamorphoses, et rythmer la tension. D'assistant, complice du masque, Le Doozi devient un subversif en combattant de l'intérieur, le pouvoir du roi auquel il appartient. Le Doozi est une figure emblématique qui pose un acte majeur de libération. Ses capacités de métamorphoses, ses prises de position courageuses notamment pendant le conseil impérial le hissent au rang de héros, de personnage extraordinaire, redresseur de torts à l'image de son mythique ancêtre Djergbeugbeu. Le Doozi acquiert une dimension sociale, culturelle, politique et transcendantale matérialisée par sa transformation en une jeune fille. Cette métamorphose acte la seconde figuration du Doozi.

La construction et l'évolution de deux personnages vont constituer un levier entre les mains du dramaturge ivoirien pour la mise en œuvre d'un projet artistique ayant pour centre d'intérêt Le Doozi. Il s'agit du Doozi lui-même et de Niobé dans Le secret des dieux. En effet, il faut le rappeler, Le Doozi est un danseur qui peut de ce fait revêtir la symbolique rythmique. Son origine sacrée peut aisément permettre de l'inscrire dans une posture spirituelle, dans une dimension métaphysique. Par ailleurs, sa connaissance du monde politique lui permet d'endosser le rôle d'homme de pouvoir à la pointe des luttes décisives. Cette triple représentation du Doozi devient un prétexte pour la fabrication d'un personnage récurrent pouvant transcender les époques et les espaces à l'image des héros mythiques antiques comme Prométhée, Hyppolyte ou des héros mythiques modernes réincarnés dans une certaine mesure au cinéma sous les traits de superman et de superwoman. Quant à Niobé, elle porte un nom d'origine mythique. Elle est l'orgueilleuse fille de Tantale et épouse d'Amphion dans la mythologie grecque qui a été punie par les dieux pour s'être vantée de sa fécondité. Zadi Zaourou ne retient de ce personnage que la force de caractère qui le pousse à se rebeller contre les dieux. Aussi, Niobé, jeune étudiante révoltée, leader du mouvement populaire naissant emprunte-t-elle également les traits caractéristiques du héros extraordinaire capable de mener des exploits prodigieux tout à l'image du héros du didiga ancien. Zadi Zaourou fait évoluer ces deux personnages, d'abord de façon parallèle dans deux espaces différents, tel qu'indiqué au tableau rythmique V ; 
puis les fait effectivement se rencontrer au tableau II dans une étrange atmosphère après que Le Doozi a été vu métamorphosé en jeune fille. Cette transfiguration est le signe d'un transfert ou d'une incarnation symbolique matérialisée par la rencontre physique symbole de nouement d'un pacte de ralliement. La métamorphose du Doozi devient le symbole d'une identification à la jeune fille et à son combat. Le lecteur spectateur averti apercevra ainsi Le Doozi dans les tribulations de Shéhérazade, la conteuse engagée dans la lutte pour la liberté des femmes ; dans les métamorphoses de Mahié, la guerrière dont l'androgynie symbolique peut se lire comme une métaphore de la société et du pouvoir ; dans le rôle de l'arc musicale, maître d'initiation du néophyte dans le cercle de jeu mais également dans celui de l'initié combattant contre le monarque et ses esprits.

Le Doozi s'oppose à Edoukou-roi tout autant que Niobé. Zadi Zaourou opère une transformation physique du Doozi en une jeune fille et jette un pont entre les deux personnages. Ce lien sacré devient un espace perméable dans lequel le dramaturge ivoirien fait voyager Le Doozi. Il devient selon les circonstances et en plus de Niobé, Shéhérazade, Mamie Wata, Zouzou, Mahié, l'arc musical, l'Initié, tous, des figures diverses du héros antique Djergbeugbeu, confronté à une intrigue relevant toujours de l'impensable et dont Le Doozi est le symbole le plus abouti. Le Doozi est donc à la fois, humain et esprit, homme et femme, initié et en initiation, masque et accompagnateur du masque, chasseur et conteur, agent rythmique et danseur, en définitive, «l'unité parfaite de chiffre $7 ; 7=1$, Didiga! » (La guerre des femmes, p. 69.) Par la transformation physique, la mise en scène symétrique, la rencontre physique, Zadi Zaourou modélise la pérennisation du personnage du Doozi qui sera constamment réincarné dans tout le système initiatique du didiga.

\section{La liminalité comme essence du didiga}

Bien que le terme liminalité soit forgé par Victor Turner, la théorie de la liminalité tire son origine des travaux d'Arnold Van Gennep sur les rites de passage. Ses réflexions ont pour point de départ les crises de la vie dont tout être humain peut se servir pour apporter sa contribution à l'édification de la société. La liminalité désigne un état de l'entre-deux, c'est-à-dire le moment où un individu perd un premier statut sans pour autant accéder au second. C'est une phase transitoire pareille à celle dans laquelle se trouve l'initié. Dans la phase préliminaire, l'individu se sépare du monde antérieur afin d'entamer les rites liminaires constitués d'épreuves à franchir pour mériter la reconnaissance de 
sa communauté d'origine. Cette dernière phase postliminaire se réalise dans la société d'où part l'initié car c'est elle qui constate les changements opérés dans sa vie. Réintégrer le groupe explique pourquoi, il faut achever l'initiation pour changer de statut. L'expérience initiatique a donc pour but d'accéder à un autre statut, entendu comme un rite de passage tel que défini par Arnold Van Gennep : « Pour les groupes, comme pour les individus, vivre c'est sans cesse se désagréger et se reconstituer, changer d'état et de forme, mourir et renaître. C'est agir puis s'arrêter, attendre et se reposer, pour recommencer ensuite à agir, mais autrement ${ }^{15}$. » La théorie de la liminalité est donc au cœur de la question de l'initiation qui devient un temps d'introspection, un espace de remise en cause. Le didiga de Zadi Zaourou s'interroge sur l'essence de l'art et de l'existence humaine et se déploie dans une telle vision :

La guerre des Femmes est un «Didiga», c'est-à-dire un théâtre à vocation initiatique. [...] Comme nos contes et comme nos mythes qui lui ont fourni l'essentiel de sa matière et de ses formes, comme nos grands rituels, elle est un mode de questionnement aussi bien pour l'acteur qui l'interprète que pour le public qui la découvre. [...] Le fondement du didiga c'est l'initiation dans la mesure où la progression dramatique d'un didiga sera toujours un parcours initiatique ${ }^{16}$.

L'initiation constitue le noyau central du didiga. Le Doozi est la figure symbolique de l'initié dans le didiga. Il devient par conséquent l'être liminal dont le parcours initiatique structure la progression dramatique du didiga. Deux cercles d'initiation vont servir d'éléments d'analyse de la liminalité du Doozi comme essence du didiga : le cercle d'arc musical dans La Termitière et le cercle de conte dans Le secret des dieux.

Le cercle d'arc musical avec comme personnages l'arc musical ou le dôdô, le joueur-initié et les spectateurs en représentation est un cercle de jeu qui fonctionne comme une mise en abyme, un théâtre dans le théâtre. Ce jeu rappelle le prologue de La tragédie du roi Christophe d'Aimé Césaire, lui-même maître à penser de Zadi Zaourou. Le combat des deux coqs dans la gagaire représente la lutte politique opposant Christophe à Pétion et symbolise tout le destin tragique de Haïti. Le jeu de la graine est donc une mise en abyme qui a les mêmes fonctionnalités que le combat des cops. L'entrée en scène théâtrale du joueur et de ses partisans peut renvoyer à la fin des épreuves d'initiation et au retour de l'initié dans sa communauté. Le jeu renvoie dès lors à la métaphore de l'initiation dans la mesure où la dimension théâtrale du jeu peut se lire comme une forme d'autoreprésentation dans le sens de représentation dédoublée. Le didiga se représente lui-même en tant qu'art de la scène. Dans le didiga, il y a un jeu permanent, reflet de la vie elle-même cadre expérimental 
de l'existence et tout le sens de l'initiation. Les différents timbres de voix de l'arc qui rythment la progression dramatique fonctionnent sans doute en lien avec le rythme à trois pulsations (La termitière p. 75) et la description du rôle du Doozi (Le secret des dieux, p. 154). L'arc musical accompagne ainsi «l'initié des initiés » chaque fois une situation de crise interpelle sa conscience.

Dans Le secret des dieux, le prologue est un cercle de conte qui met en scène deux récitants sur fond d'arc musical. Le premier récitant et le second prennent la parole de façon alternée au milieu du cercle formé par le public.

PREMIER RÉCITANT : C'était aux époques révolues des guinns et des sartiyis. C'était aux époques des méfaits de Fontidoua. Les femmes portaient encore la barbe et les brebis terrifiaient lions et lionceaux, hyènes et guépards, la jungle vivant sa férique adolescence.

DEUXIÈME RÉCITANT : Il n'y avait encore ni masque ni maître d'initiation. L'homme connaissait pourtant déjà le vertige des temps d'avant les temps, le tourbillon sacré qui l'égare aujourd'hui. (Prologue, p. 20.)

Le premier récitant présente le cadre mythique du récit. Le deuxième récitant prend la parole à son tour mais situe le récit dans un contexte actuel. Toute la séquence de prise de paroles se déroule sur ce mode qui permet au second d'expliciter, d'actualiser, de ponctuer les propos du premier. Locha Mateso présente ce noyau rythmique en ces termes : «Dans le cercle de conte, deux personnages prennent la parole à tour de rôle : l'épicentre et le conteur. Le rôle de l'épicentre est de rythmer la parole du conteur ${ }^{17}$. $\gg$ Cette organisation s'établit autour du conteur accompagné dans le cercle de conte par l'épicentre. Zadi Zaourou a appelé cette situation de prise de paroles, la circulation de la parole à trois et identifié le deuxième récitant ou l'épicentre comme étant l'agent rythmique.

Ce prologue a servi de mise en train, de warming up dans la mesure où après avoir planté le décor, ces deux personnages ont disparu au profit du Doozi. En effet, Le secret des dieux se présente comme un conte entamé dans le prologue et continué par le biais des tableaux rythmiques, de certaines didascalies narratives mais surtout, à travers Le Doozi dans le rôle de l'agent rythmique. Au tableau III, l'empereur Edoukou charge le conteur Katangbo de conter l'histoire de la chèvre de monsieur Seguin relativement à l'ingratitude des jeunes révoltés qui constituent le public avec Edoukou et ses ministres.

L'EMPEREUR : Raconte à ces gens l'histoire de la Chèvre de Monsieur Séguin. (Et Katangbo dans une langue africaine raconte l'histoire accompagné du Doozi 
qui lui sert d'agent rythmique pour la traduction en français des temps forts de cette histoire.) (Tableau III, p. 92.)

Pour rappel, Le Doozi a également un statut de ministre mais, il joue dans ce tableau, le rôle d'agent rythmique chargé de relayer un conte. Cependant, lors de la séquence qu'on pourrait appeler aujourd'hui un debriefing, Le Doozi raille les idées émises par ses interlocuteurs.

L'IMPÉRATRICE : Je te l'avais dit. Ils sont fous. Complètement fous ! C'est l'asile qu'il leur faut; non la vallée. (Tableau III, p. 100)

Le Doozi saisit cette occasion pour s'en prendre à la famille impériale et laisse sous-entendre les bouleversements à venir, trahissant ainsi son secret, celui d'avoir rejoint les rangs de l'opposition. Le Doozi est sans doute déjà dans cette phase magico-religieuse de flottement.

LE DOOZI (riant) : Les fous ne sont peut-être pas où vous croyez ! C'est vrai qu'il viendra pour sûr... (Un temps) Il viendra. (Tableau III, p. 100)

Contrairement au deuxième récitant du prologue, Le Doozi n'est ni un répétiteur ni un modérateur sans opinion, il montre que le rôle d'agent rythmique a évolué avec le temps pour prendre une dimension révolutionnaire compte tenu du contexte politique nouveau. Edoukou est un dictateur qui opprime son peuple. Les jeunes avec à leur tête Niobé tentent de bouleverser l'ordre établi pour que naisse un nouvel ordre politique. Le Doozi comprend que le moment est venu de passer à autre chose, de surmonter d'autres épreuves et d'entamer d'autres combats. Il trouve un nouveau sens à sa vie en changeant de statut pour devenir opposant. Le Doozi est l'être liminal, l'être en transit dans la mesure où il change constamment de statut. D'assistant du masque fidèle et serviable, il devient un serviteur de l'État en tant que ministre. Mais ce pouvoir auquel il a appartenu a failli. Il devient urgent de s'en détacher. Dans cette arène politique où les dignitaires s'accrochent à leurs privilèges, Le Doozi tourne la page pour revêtir les habits d'un opposant farouche au pouvoir. Le lecteur spectateur le découvre ainsi en train de haranguer les foules comme pour passer le test d'aptitude pour l'entrée en opposition au tableau II. Avec la construction du personnage du Doozi, Zadi Zaourou redessine le cycle de la vie tel que théorisé par Arnold Van Gennep. L'être humain est permanemment en initiation, en quête de régénérescence. C'est un cycle sans fin.

Le théâtre de Zadi Zaourou est un théâtre d'initiation, par extension, un théâtre métaphysique. Dans la phase d'initiation, l'initié n'a pas de contact 
direct avec le maître d'initiation en général. Il passe par des esprits, la mère défunte. Ce qui crée le décalage à trois, la triangulation de la communication. Intégrer l'agent rythmique fonctionnant dans un espace triadique, entre l'émetteur et le récepteur et jouant le rôle d'accompagnateur du conteur principal, le hisse au rang d'un potentiel conteur donc d'un personnage en initiation. Au niveau de la conception transcendantale du monde, l'agent rythmique devient le pôle trois, c'est-à-dire le prêtre situé entre la transcendance et les hommes ordinaires. Tout le monde ne peut pas percevoir le discours de la transcendance. Il y a pour ainsi dire, des agents à partir desquels la transcendance va communiquer avec les humains non-initiés. Dans la conception du didiga, il y a toujours une dimension métaphysique de la vie. Cette récurrence de l'agent rythmique dans le didiga s'explique donc par une conception métaphysique du monde. La théâtralisation de la tripartition de l'espace de communication dans le didiga révèle la pensée profonde du dramaturge. Zadi Zaourou s'oppose en réalité à une lecture complètement matérielle du monde. C'est là un des grands paradoxes de l'homme : comment concevoir le didiga et enseigner le matérialisme dialectique. C'est impensable, c'est du didiga.

\section{Conclusion}

Cette analyse a montré que le didiga nouveau prend appui sur le didiga ancien pour entrer en dialogue avec différentes expressions artistiques. Le Doozi personnage caractéristique du didiga se construit et évolue au gré des situations socio-politiques. Être liminal par excellence, Le Doozi est la métaphore du didiga. Les différentes figurations du Doozi manifestent sa permanence physique et métaphorique. La démarche de Zadi Zaourou est simple : utiliser les formes de représentations artistiques qui s'apparentent au théâtre pour inventer un art dramatique nouveau. Le didiga a de ce point de vue été une belle occasion de renouvellement du théâtre africain moderne. Même si, le didiga est apparu à un moment comme un art sans perspective, il faut relever qu'il constitue aujourd' hui une source d'inspiration pour des dramaturges dont Liazéré et Tiburce Koffi.

\section{Bibliographie}

Aristote, Poétique, Paris, Société d’Éditions Les Belles Lettres, 1969.

Aron Paul, Saint-Jacques Denis, Viala Alain (dir.), Le dictionnaire du Littéraire, Paris, Presses Universitaires de France, 2002. 
Biet Christian et Triau Christophe, Qu'est-ce que le théâtre?, Paris, Gallimard, 2006. Césaire Aimé, La tragédie du roi Christophe, Paris, Présence Africaine, 1970.

Dadié Bernard, "Mon pays et son théâtre ", in Assémien Debylé roi du Sanwi, Abidjan, CEDA, 1979.

Koffi Tiburce, Mélédouman suivi de pour un casting, Abidjan, NEI/CEDA, 2014.

Kotchy N'Guessan Barthélemy, Éléments culturels et formes de repré-sentation en Afrique noire, l'exemple de la Côte d'Ivoire, t. II, Thèse de doctorat d'État, Université de Paris VIII Vincennes Saint Denis, 1983.

Lehmann Hans-Thies, Le théâtre postdramatique, Paris, L'Arche Éditeur, 2002. Liazéré, La complainte d'Ewadi, Carnières-Morlanwelz, Édition Lansman, 1996.

Mateso Locha, La littérature africaine et sa critique, Paris, A.C.C.T. et Karthala, 1986.

Médéhouégnon Pierre, Le théâtre francophone de l'Afrique de l'Ouest des origines à nos jours, Jéricho-Cotonou, CAAREC Éditions, 2010.

Mémel Harris Foté, «L'anthropologie du théâtre négro-africain traditionnel », in Actes du colloque sur le théâtre négro-africain, Paris, Présence Africaine, 1971.

Pavis Patrice, Dictionnaire du théâtre, Paris, Dunod, 1996.

Sedar Senghor Léopold, Négritude et Humanisme, Paris, Seuil, 1984.

Touré Aboubakar Cyprien, La griotique mémoires et réflexions, Paris, L'Harmattan, 2014.

Van Gennep Arnold, Les rites de passage : Étude systématique des rites, Paris, Librairie Critique Emile Nourry, 1909.

Wondji Christophe, "Chanson et culture populaire en Côte d'Ivoire », in La chanson populaire en Côte d'Ivoire, Paris, Présence Africaine, 1986.

Zadi Zaourou Bottey, Le secret des dieux, Torino, L'Italia La Rosa Éditrice, 1999.

— L La guerre des femmes suivie de La termitière, Abidjan, NEI-Neter, 2001.

, "Le mythe, le prêtre et le poète : puissance unifiante du rythme », in Colloque sur littérature et esthétique négro-africaines, Abidjan-Dakar, Les Nouvelles Éditions Africaines, 1979.

\section{Notes}

1. Aron Paul, Saint-Jacques Denis, Viala Alain (dir.), Le dictionnaire du Littéraire, Paris, Presses Universitaires de France, 2002, p. 588.

2. Patrice Pavis, Dictionnaire du théâtre, Paris, Dunod, 1996, p. 336.

3. Bernard Dadié, "Mon pays et son théâtre ", in Assémien Dehylé roi du Sanwi, Abidjan, CEDA, 1979.

4. Aron Paul, op. cit, p. 588.

5. Aristote, Poétique, Paris, Les Belles Lettres, 1969, p. 28.

6. Christian Biet et Christophe Triau, Qu'est-ce que le théâtre?, Paris, Gallimard, 2006, p. 7.

7. Bottey Zadi Zaourou, La guerre des femmes suivie de La termitière, Abidjan, NEI-Neter, 2001, Postface, p. 124. 
8. Ibid., p. 123.

9. Léopold Sédar Senghor, Négritude et Humanisme, Paris, Seuil, 1984, p. 209.

10. Harris Memel-Fotê, « L'anthropologie du théâtre négro-africain traditionnel » in Actes du colloque sur le théâtre négro-africain, Paris, Présence Africaine, 1971, p. 25-30.

11. Christophe Wondji, "Chanson et culture populaire en Côte d'Ivoire ", in La chanson populaire en Côte d'Ivoire, Paris, Présence Africaine 1986, p. 11.

12. Zadi Zaourou, "Le mythe, le prêtre et le poète : puissance unifiante du rythme », in Colloque sur littérature et esthétique négro-africaines, Abidjan-Dakar, Les Nouvelles Éditions Africaines, 1979, p. 118.

13. Kotchy, N'Guessan Barthélemy, Éléments culturels et formes de représentation en Afrique noire, l'exemple de la Côte d'Ivoire, t. II, Thèse de doctorat d'État, Université de Paris VIII Vincennes Saint Denis, 1983.

14. Zadi Zaourou Bottey, Le secret des dieux, Torino, L'Italia La Rosa Editrice, 1999, p. 154.

15. Arnold Van Gennep, Les rites de passage : Étude systématique des rites, Paris, Librairie Critique Emile Nourry, 1909, p. 272.

16. Bottey Zadi Zaourou, op. cit., p. 129.

17. Locha Mateso, La littérature africaine et sa critique, Paris, A.C.C.T./Karthala, 1986, p. 316. 